\title{
Malaria therapy: where do we stand, what next?
}

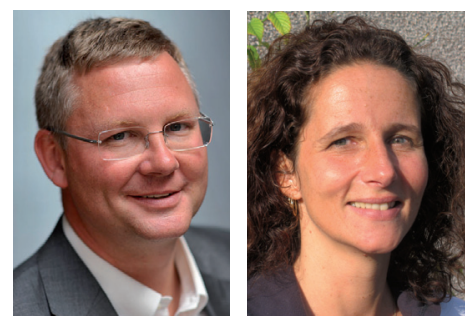

"Whilst the economic burden of the

disease remains huge, estimates

suggest that current morbidity

and mortality have reached

a historical low"

Martin Peter Grobusch ${ }^{\dagger 1,2,3,4}$ \& Michèle van Vugt ${ }^{1,2}$

IInfectious Diseases, Tropical Medicine \& AIDS, Academic Medical Center, University of Amsterdam,

P.O. Box 22700, 1100 DE Amsterdam, The Netherlands

${ }^{2}$ Amsterdam Institute for Global Health \& Development (AIGHD), Amsterdam, The Netherlands

${ }^{3}$ Institute of Tropical Medicine, University of Tübingen, Germany

${ }^{4}$ Division of Infectious Diseases, Department of Medicine, Faculty of Health Sciences, University of the

Witwatersrand, Johannesburg, South Africa

†Author for correspondence: Tel. : +31 205664380 = Fax: +31 206972286 - m.p.grobusch@amc.uva.nl

With the recent unprecedented boosts to the development of novel, and optimized use of existing, malaria control tools the malaria world continues to change rapidly [1]. Whilst half of the global population remains at risk of malaria and the economic burden of the disease remains huge, estimates suggest that current morbidity and mortality (with approximately $250 \mathrm{mil}$ lion cases and 860,000 deaths, the majority being children in sub-Saharan Africa) have reached a historical low [2]. The recently published second edition of the WHO's Guidelines for the Treatment of Malaria [3] accounts for novel developments in the field of treatment with artemisinin-based combination therapies (ACTs) now including dihydroartemisinpiperaquine [4] as an option to treat uncomplicated falciparum malaria [5] and considering ACTs with long half-life partners such as mefloquine for the treatment of chloroquine-resistant vivax malaria [6]. The guidelines emphasize appropriate case management, such as to treat for malaria only after diagnosis by means of rapid diagnostic 'dipstick' testing (RDT) in places where reliable light microscopy cannot be performed; and to base treatment on clinical suspicion only if parasitological diagnosis is inaccessible.

Whereas a range of appropriate and affordable ACTs and improved pediatric formulations thereof [7] (and non-artemisinin-containing treatment alternatives where indicated) are at hand in principle for first-line treatment of uncomplicated falciparum malaria treatment in endemic areas (with artesunate as a backbone drug to be used in preference to quinine for severe falciparum malaria), manufacturing standards and availability of GMP (good manufacturing practice) products continue to pose a significant problem both in routine use and in clinical research. This results in a bottleneck regarding availability of some high quality artemisin-based drugs. The WHO is addressing this urgent problem and has developed a tool providing guidance for the implementation of acceptable ACT production and procurement standards to increase the availability of GMP products [8].

Gains in the fight against malaria threaten to be off-set, at least in part and in the medium term, by growing evidence of artemisin resistance - despite of a considerable antimalarial drug pipeline [101], it is estimated that it will take at least another decade before a new class of antimalarials will reach the market [8]. First reported from the Thai-Cambodian border region (the hotbed of antimalarial drug resistance development) and later confirmed in studies from western Cambodia and adjacent areas [9-12], there is growing evidence of emerging reduced susceptibility of Plasmodium falciparum against artemisinins. The molecular mechanism of action continues to be unclear [13] and molecular markers of resistance to date remain, by-and-large, unidentified [14]. In order to identify other foci of potential emergence of artemisinin derivative resistance and to accelerate the identification and validation of molecular markers of parasite resistance, the Worldwide Antimalarial Resistance Network has called for the development of an artemisinin resistance marker platform [102]. Aside from the development of 'true' plasmodial resistance against artemisinins, the 'human factor', of counterfeit drugs being introduced into the markets is a well-recognized yet poorly

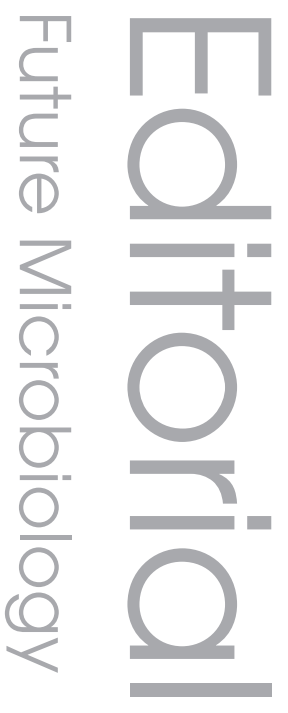

\section{Keywords}

- artemisinin-based

combination therapies

- good manufactuirng

practice $=$ malaria $=$ rapid

diagnostic test $=$ treatment

- WHO guidelines

future medicine $^{\text {poss }}$ fs 


\section{"Gains in the fight against malaria threaten to be off-set ... by growing evidence of artemisin resistance."}

controllable problem. This additional problem contributes to treatment failure, development of drug resistance, economic waste [15] and possibly flawed clinical study results [16]. To date, counterfeit artemisinin derivatives and ACTs have been described in at least six countries throughout Africa [15].

Which other major challenges are we facing on the long and winding road to improved malaria control, let alone eradication? Chuma and colleagues [17] reviewed the literature regarding access to prompt and effective malaria treatment in Kenya and concluded that despite increased efforts, the majority of patients still lack prompt access to effective treatment - one reason is, and remains, the distance from a healthcare facility [17] - this is a logistic and household-specific economic problem, which can hardly be resolved for many living in rural areas in developing countries. However, the main observation is that the informal health sector (referring to drug sellers/shopkeepers that do not require a license to sell over-thecounter drugs yet may be selling prescription drugs illegally) in Kenya continue to receive vast numbers of malaria treatment-seeking customers. According to their findings, the proportion of over-the-counter treatments may exceed $50 \%$ of the total in some settings - which suggests that those $50 \%$ would not benefit at all from improvements made to changing official treatment strategies with ACTs following RDT, whereas many of our projections on progress in malaria control hinge on the assumption that whatever policy is implemented, it is going to somehow reach those in need.

Maslove and colleagues systematically reviewed qualitative studies examining knowledge, attitudes and practices of people living in malaria-endemic regions of sub-Saharan Africa and conclude that the cultural and social contexts in which malaria prevention and treatment program are deployed must be accounted for in order to suceed [18], probably on a far larger scale and more tailored to the individual situation than seems to currently be the case. Kokwaro, looking at the various challenges in the management of malaria, stresses that the (ongoing) education of health workers and communities is vital to control programs [19]. In order to further change perceptions on malaria within communities, to inform appropriately about the technicalities of diagnosis and treatment, particularly in remoter areas, and to allow access to malaria control tools, from vector control tools to preventive treatment of high-risk groups and diagnosis followed by treatment, novel routes of assisting people in need gaining access to malaria control tools are needed.

One route that remains underexplored so far, may be to strengthen community health worker systems. A recent study from Ethiopia showed that ACT deployment in combination with RDT through a community-based service in a remote rural population reduced malaria transmission, lowered the case burden on established health facilities and reduced malaria morbidity and mortality [20]; a concept which needs to be studied further in various settings. Outreach programs relying on well-trained community health workers may, in certain settings, help to assist in reducing the magnitude of some of the aforementioned problems, particularly if linked to reference healthcare facilities.

\section{Financial \& competing interests disclosure \\ The authors have no relevant affiliations or financial involvement with any organization or entity with a financial interest in or financial conflict with the sub- ject matter or materials discussed in the manuscript. This includes employment, consultancies, honoraria, stock ownership or options, expert testimony, grants or patents received or pending, or royalties. \\ No writing assistance was utilized in the production of this manuscript.}

\section{Bibliography}

1. Grobusch MP, Schellenberg D: The rapidly changing world of malaria: where do we stand, and where do we go from here? Therapy 7(1), 1-2 (2010).

2. World Health Organization: World Malaria Report 2009. WHO Press, Geneva, Switzerland (2009).

3. World Health Organization: Guidelines for the Treatment of Malaria (2nd Edition). WHO Press, Geneva, Switzerland (2010).
4. D'Alessandro U: Progress in the development of piperaquine combinations for the treatment of malaria. Curr. Opin. Infect. Dis. 22(6), 588-592 (2009).

5. Sinclair D, Zani B, Donegan S, Olliaro P, Garner P: Artemisin-based combination therapy for treating uncomplicated malaria. Coch. Database Syst. Rev. 8(3), CD007483 (2009).

6. Douglas NM, Anstey NM, Angus BJ, Nosten F, Price RN: Artemisinin combination therapy for vivax malaria. Lancet Infect. Dis. 10, 405-416 (2010).
7. World Health Organization: Good Procurement Practices for Artemisin-Based Antimalarial Medicines. WHO Press, Geneva, Switzerland (2010).

8. Kurth F, Bélard S, Adegnika AA, Gaye O, Kremsner PG, Ramharter M: Do pediatric drug formulations of artemisinin combination therapies improve the treatment of children with malaria? A systematic review and meta-analysis. Lancet Infect. Dis. 10, 125-132 (2010). 
9. Denis MB, Tsuyuoka R, Lim P et al.: Efficacy of artemether-lumefantrine for the treatment of uncomplicated falciparum malaria in northwest Cambodia. Trop. Med. Int. Health 11(12), 1800-1807 (2006).

10. Noedl H, Se Y, Schaecher K, Smith BL, Socheat D, Fukuda MM: Evidence of artemisin-resistant malaria in Western Cambodia. N. Engl. J. Med. 359 (24), 2619-2620 (2008).

11. Noedl H, Socheat D, Satimai W: Artemisinin-resistant malaria in Asia. N. Engl. J. Med. 361(5), 540-541 (2009).

12. Dondorp AM, Nosten F, Yi P et al.: Artemisinin resistance in Plasmodium falciparum malaria. N. Engl. J. Med. 361(5), 455-467 (2009).

13. O'Neill PM, Barton VE, Ward SA: The molecular mechanism of action of artemisin - the debate continues. Molecules 15(3), 1705-1721 (2010).
14. Dondorp AM, Yeung S, White L: Artemisin resistance : current status and scenarios for containment. Nature Rev. Microbiol. 8, 272-280 (2010).

15. Kaur H, Green MD, Hostetler DM, Fernandez FM, Newton PN: Antimalarial drug quality: methods to detect drugs. Therapy 7(1), 49-57 (2010).

16. Giha HA: Artemisinin derivatives for treatment of uncomplicated Plasmodium falciparum malaria in Sudan: too early for too much hope. Parasitol. Res. 106, 549-552 (2010).

17. Chuma J, Abuya T, Memusi D: Reviewing the literature on access to prompt and effective malaria treatment in Kenya: implications for meeting the Abuja targets. Malaria J. 8, 243 (2009).

18. Maslove DM, Mnyusiwalla A, Mills EJ: Barriers to the effective treatment and prevention of malaria in Africa: a systematic review of qualitative studies. BMC Intern. Health Human Rights 9, 26 (2009).
19. Kokwaro G: Ongoing challenges in the management of malaria. Malaria J. 8(Suppl. 1), S2 (2009).

20. Lemma H, Byass P, Desta A et al.: Deploying artemether-lumefantrine with rapid testing in Ethiopian communities: impact on malaria morbidity, mortality and healthcare resources. Trop. Med. Int. Health 15(2), 241-250 (2010).

\section{Websites}

101. Medicine for Malaria Venture: Interactive Project Portfolio www.mmv.org/research-development/ project-portfolio (Accessed 19 July 2010)

102. Artemisinin Resista nce Marker (ARM) Platform - call for participants www.wwarn.org/arm (Accessed 15 July 2010) 